\title{
Evaluation of magnetic resonance imaging findings in adhesive capsulitis: which quantitative findings are most valuable?
}

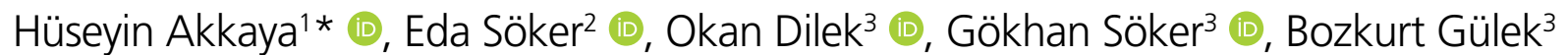

\section{SUMMARY}

OBJECTIVE: This study aims to investigate magnetic resonance imaging (MRI) findings in adhesive capsulitis (AC) and determine the most valuable MRI finding in diagnosis using easily applied quantitative methods.

METHODS: Shoulder MRI was performed on 193 patients who were diagnosed with AC by clinical examination and 116 controls. Axillary pouch thickness (APT), superior and inferior glenohumeral ligament (SGHL and IGHL) thickness, coracohumeral ligament (CHL) thickness, fluid increase and soft-tissue thickness in the rotator interval (RI), and increases in the fluid and signal in the localization of biceps tendon attachment were evaluated. MRI examinations were assessed by three radiologists blinded to the clinical findings of the patients, and the results were obtained based on consensus and records.

RESULTS: There were 119 women and 74 men in the $A C$ group and 80 women and 36 men in the control group. IGHL, SGHL, RI, and $\mathrm{CHL}$ thicknesses were measured thicker in $\mathrm{AC}$ patients than in the control group. When $\mathrm{IGHL}=4, \mathrm{RI}=3.6, \mathrm{SGHL}=2.0, \mathrm{CHL}=4.6-\mathrm{mm}$ cutoff, the area under the receiver operating characteristic (ROC) curve (AUC) values were $0.700,0.922,0.972$, and 0.783 , respectively. CONCLUSIONS: According to the results obtained in this study, IGHL=4 mm, RI=3.6 mm, SGHL=2.0 mm, and CHL=4.6 mm can support the diagnosis of AC. Using the quantitative values in diagnosis can provide objective criteria and prevent variability among interpreters. KEYWORDS: Adhesive capsulitis. Pain. Shoulder. Frozen shoulder. Musculoskeletal diseases. Magnetic resonance image.

\section{INTRODUCTION}

Adhesive capsulitis (AC) is a clinical condition of uncertain etiology. Diagnosis is mostly clinical, and the role of radiology is mostly secondary to confirm or deny the presence of contributory causes and perhaps the extent of disease ${ }^{1}$. AC is a diffuse inflammatory disease involving the scapulohumeral joint, joint capsule, joint synovial tissue, glenohumeral ligaments, periarticular tendon and bursae (especially the subacromial bursa), and biceps tendon sheath ${ }^{2}$.

There are various studies on the magnetic resonance imaging (MRI) findings of AC, examining signal changes and morphological changes in different anatomical structures in the shoulder joint ${ }^{3-5}$. However, a clear consensus has not yet been achieved among the findings of studies on the diagnosis of this condition. Therefore, interobserver variability among physicians interpreting MRI may be very high. This study aimed to quantitatively evaluate the changes in the MRI signal and morphological changes including the thickness of the capsuloligamentous structures and considered to be affected by AC and to compare the diagnostic values of these findings.

\footnotetext{
'Siverek State Hospital, Department of Radiology - Siverek, Turkey.

${ }^{2}$ University of Health Sciences, Adana Teaching and Research Hospital, Department of Physical Medicine and Rehabilitation - Adana, Turkey.

3University of Health Sciences, Adana Teaching and Research Hospital, Department of Radiology - Adana, Turkey.

*Corresponding author: dr.hsynakkaya@gmail.com

Conflicts of interest: the authors declare there is no conflicts of interest. Funding: none.

Received on September 06, 2021. Accepted on September 06, 2021.
} 


\section{METHODS}

This retrospective study was approved by our institutional ethical committee and carried out in accordance with the Declaration of Helsinki and the Good Clinical Practice Guidelines. The requirement for informed consent from the patients was waived due to the retrospective nature of the study.

Patients who were diagnosed with AC at the Physical Therapy and Rehabilitation Clinic between January 2018 and April 2020 and underwent a shoulder MRI examination were included in this study. The diagnosis of AC was made by the clinician based on the clinical examination. The control group was formed with patients that were referred to MRI by the same clinic for the exclusion of conditions other than AC, such as fibromyalgia and myofascial pain syndrome, and were determined to have no morphological and signal changes in the structures affected by AC. The patient and control group list was determined by the clinician. The radiologists who performed the evaluation had no clinical knowledge of the patients.

MRI examinations were performed using a $1.5 \mathrm{~T}$ device (Philips Ingenia, Best, The Netherlands) and a phased array shoulder dedicated coil. A 160-mm field of view (FOV), 3-mm slice thickness, $0.3-\mathrm{mm}$ interslice gap, and 256×256 matrix were used for all sequences. The parameters used for the oblique coronal proton density (PD) sequence were TR/TE 3,700/30 ms, for the oblique coronal T1W sequence were 608/9 ms TR/TE, for the axial PD sequence were TR/TE 4,200/30 ms, for the oblique sagittal PD sequence were TR/TE 4,600/25 ms, and for the sagittal T1W sequence were TR/TE 608/9 ms.

\section{MRI evaluation}

All images evaluated were non-contrast shoulder MRIs taken with the same device. Fluid increase and soft-tissue thickness in the rotator interval (RI) were shown in oblique sagittal PD images. The presence of increased fluid and signal at the attachment site of the biceps tendon was shown in oblique sagittal PD images. Coracohumeral ligament (CHL) thickness and obliteration of the subcoracoid fat triangle were shown in oblique sagittal T1W images (Figure 1). The study was conducted retrospectively, and the evaluation was undertaken based on the consensus of three radiologists with 30,16, and 4 years of experience in radiology, who examined the images obtained from the Picture Archiving and Communication System of our hospital.

\section{Statistical analysis}

The Statistical Package for the Social Sciences (SPSS) package program v. 23.0 was used for the statistical analysis of the data. The chi-square and Fisher's exact tests were used for the comparison of categorical variables. The Shapiro-Wilk test was conducted to determine whether the parameters investigated in this study showed a normal distribution. In the comparison of continuous measurements between the groups, the normality assumption was checked, and the independent Student's $t$-test was used for normally distributed parameters while the Mann-Whitney $U$ test was used for the parameters that did not conform to a normal distribution. The statistical significance level was accepted as 0.05 in all tests. The areas under the receiver operating characteristic (ROC) curves (AUCs) were calculated by using the method that DeLong et al. ${ }^{6}$ found. For each reader, the highest Youden index ( $J=$ sensitivity+specificity-1) was calculated to select the optimal threshold to discriminate between, and the corresponding sensitivity, specificity, positive predictive value (PPV), and negative predictive value (NPV) were computed. AUC was calculated according to the median values for inferior glenohumeral ligament (IGHL), superior glenohumeral ligament (SGHL), CHL, and RI thicknesses (Table 1).

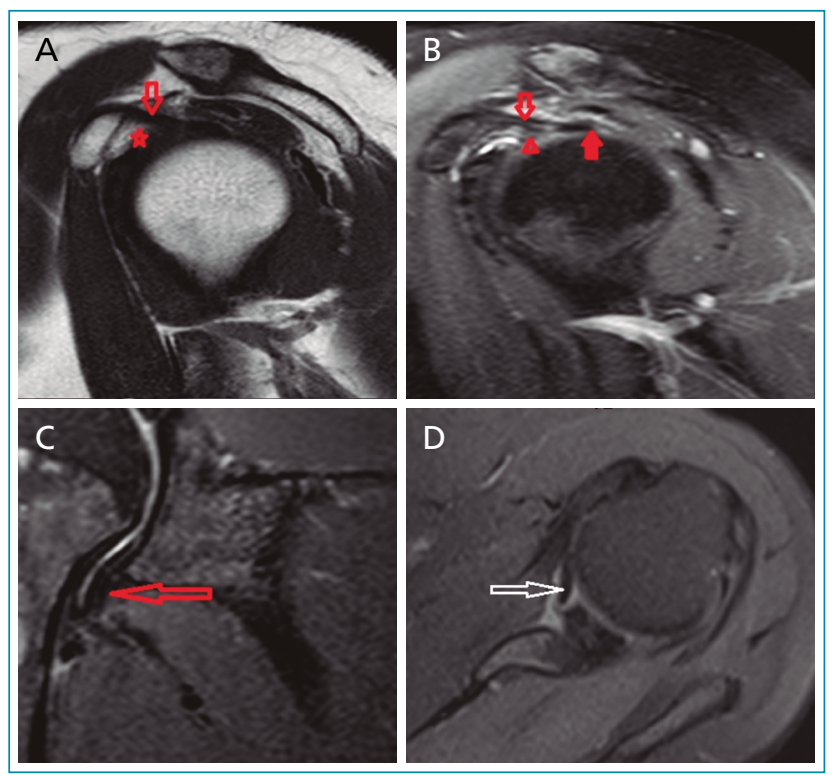

Figure 1. (A) Sagittal oblique magnetic resonance T1-weighted examination on the left shows slight thickening of the coracohumeral ligament (area marked with a hollow arrow) and mild obliteration in the subcoracoid fat tissue (marked with an asterisk). (B) Proton density-weighted sequence examination shows thickened coracohumeral ligament (area marked with a hollow arrow), fluid and soft-tissue increase in the rotator interval (area marked with an arrowhead), and signal increase at the biceps attachment point (area marked with a solid arrow). (C) In the coronal oblique plane right shoulder magnetic resonance imaging proton densityweighted sequence, thickened inferior glenohumeral ligament is seen (marked with arrow). (D) Proton density-weighted magnetic resonance imaging imaging in the axial plane of the left shoulder showed significantly thickened superior glenohumeral ligament (marked with arrow). 


\section{RESULTS}

The mean age of the AC group was $51.48 \pm 12.41$ years and that of the control group was $50.09 \pm 11.68$ years. There was no statistically significant difference between the patient and control groups in terms of age and gender ( $p$-values 0.023 and 0.194 , respectively).
There was no statistically significant difference between the two groups in terms of the obliteration of the subcoracoid fat triangle $(p=0.568)$. Concerning the evaluation of signal increase at the attachment site of the biceps tendon, there was a statistically significant difference between the two groups $(\mathrm{p}<0.05)$ (Table 2$)$.

Table 1. Cutoff values and statistical analysis of parameters evaluated in this study.

\begin{tabular}{l|c|c|c|c} 
& IGHL thickness & RI soft-tissue thickness & SGHL thickness & CHL thickness \\
\hline AUC & 0.700 & 0.922 & 0.972 & 0.783 \\
\hline $95 \% \mathrm{Cl}$ & $0.621-0.771$ & $0.868-0.959$ & $0.932-0.992$ & $0.709-0.845$ \\
\hline Cutoff & $\leq 4.0$ & $\leq 3.60$ & $\leq 2.0$ & $\leq 4.6$ \\
\hline Sensitive (\%) & 100 & 100 & 100 & 100 \\
\hline $95 \% \mathrm{Cl}$ & $59-100$ & $73.5-100$ & $47.8-100$ & $29.2-100$ \\
\hline Specificity & 53.74 & 75.18 & 95.86 & 62.25 \\
\hline $95 \% \mathrm{Cl}$ & $45.3-62$ & $67.2-82.1$ & $91.2-98.5$ & $54.0-70.0$ \\
\hline PPV & 9.3 & 25.5 & 45.5 & 5 \\
\hline NPV & 100 & 100 & 100 & 100 \\
\hline P & 0.001 & $<0.001$ & $<0.001$ & 0.001 \\
\hline
\end{tabular}

IGHL: inferior glenohumeral ligament; RI: rotator interval; SCHL: superior glenohumeral ligament; CHL: coracohumeral ligament; AUC: area under the receiver operating characteristic curve; Cl: confidence interval; PPV: positive predictive value; NPV: negative predictive value.

Table 2. Comparison of the mean values of the investigated parameters between the study groups.

\begin{tabular}{|c|c|c|c|c|c|}
\hline & & Control & Adhesive capsulitis & \multirow{2}{*}{ Total } & \multirow{4}{*}{$p$} \\
\hline & & $(n=116)$ & $(n=193)$ & & \\
\hline & & n (\%) & $\mathrm{n}(\%)$ & n (\%) & \\
\hline & & Mean \pm SD & Mean \pm SD & Mean \pm SD & \\
\hline \multirow{2}{*}{ Obliteration of the subcoracoid fat triangle } & Absent & $72(62.1)$ & $126(65.3)$ & $198(64.1)$ & \multirow{2}{*}{0.568} \\
\hline & Present & $44(37.9)$ & $67(34.7)$ & $111(35.9)$ & \\
\hline \multirow{2}{*}{$\begin{array}{l}\text { Increased fluid and/or signal at the } \\
\text { attachment site of the biceps tendon }\end{array}$} & Absent & $58(50.0)$ & $42(21.8)$ & $100(32.4)$ & \multirow{2}{*}{0.001} \\
\hline & Present & $58(50.0)$ & $151(78.2)$ & $209(67.6)$ & \\
\hline \multirow{2}{*}{ Axillary pouch thickness } & & $3.93 \pm 0.72$ & $12.17 \pm 2.09$ & $9.08 \pm 4.34$ & \multirow{2}{*}{0.027} \\
\hline & & $(3-6.1)$ & $(6.1-21.2)$ & $(3-21.2)$ & \\
\hline \multirow{2}{*}{ Inferior glenohumeral ligament thickness } & & $2.68 \pm 0.63$ & $3.79 \pm 0.66$ & $3.37 \pm 0.84$ & \multirow{2}{*}{0.034} \\
\hline & & $(1.2-4.1)$ & $(2.3-6.1)$ & $(1.2-6.1)$ & \\
\hline \multirow{2}{*}{ Rotator interval soft-tissue thickness } & & $0.84 \pm 1.03$ & $3.72 \pm 2.24$ & $2.64 \pm 2.34$ & \multirow{2}{*}{0.016} \\
\hline & & $(0-4.4)$ & $(0-9.2)$ & $(0-9.2)$ & \\
\hline \multirow{2}{*}{ Coracohumeral ligament thickness } & & $2.81 \pm 0.49$ & $4.66 \pm 1.13$ & $3.97 \pm 1.30$ & \multirow{2}{*}{0.029} \\
\hline & & $(2.1-5.2)$ & $(1-8.1)$ & $(1-8.1)$ & \\
\hline Superior glenohumeral ligament thickness & & 0.041 & 0.042 & 0.043 & 0.044 \\
\hline
\end{tabular}

SD: standard deviation. 
The mean IGHL thickness was $3.79 \pm 0.66(2.3-6.1)$ and $2.68 \pm 0.63(1.2-4.1) \mathrm{mm}$, respectively; the mean SGHL thickness was $2.58 \pm 0.99(2.8-6.7)$ and $1.33 \pm 0.25$ $(0.8-2.1) \mathrm{mm}$, respectively; the mean CHL thickness was $4.66 \pm 1.13(1-8.1)$ and $2.81 \pm 0.49(2.1-5.2) \mathrm{mm}$, respectively; and the mean soft-tissue thickness in the RI was $3.72 \pm 2.24$ $(0-9.2)$ and $0.84 \pm 1.03(0-4.4) \mathrm{mm}$, respectively, between the two groups. There was a statistically significant difference between the two groups in terms of all these parameters $(\mathrm{p}<0.05)($ Table 1$)$.

IGHL thickness, RI soft-tissue thickness, SGHL thickness, CHL thickness, AUC values that are determined according to median values, corresponding sensitivity, specificity, PPV, and NPV are shown in Table 1.

\section{DISCUSSION}

Adhesive capsulitis progresses with thickening, contraction, and adhesion of the glenohumeral capsule and shoulder joint ligaments, followed by decreased capsular enlargement ${ }^{3-5}$. Gokalp et al. ${ }^{7}$ measured axillary pouch thickness (APT) using contrast-enhanced shoulder MRI and compared it between the $\mathrm{AC}$ and control groups, noticing the presence of a significant difference. However, Petchprapa et al. ${ }^{8}$ reported that while there was no difference in the axillary pouch width between the patients with $\mathrm{AC}$ and the control group, the capsule-synovium thickness in the axillary pouch was increased in the former. Sofka et al. ${ }^{9}$ showed that the APT ranged from 2 to $13 \mathrm{~mm}$ among these patients. In this study, using non-contrast shoulder MRI, the APT was measured as $12.17 \pm 2.09(6-21) \mathrm{mm}$ for the AC group and $3.93 \pm 0.72(3-6) \mathrm{mm}$ for the control group, and the difference between the two groups was statistically significant. However, it is noteworthy that in cases with suspected $\mathrm{AC}$, the thickness of the axillary pouch increased compared with the controls. In this respect, considering the APT when evaluating shoulder MRI in patients with suspected AC will be useful for diagnosis.

In this study, the difference between the two groups in terms of signal changes at the biceps tendon attachment site was statistically significant $(p<0.05)$. These findings are consistent with those reported by previous studies. However, the presence of an increased signal at the attachment site of the biceps tendon can be seen in many synovitis and synovitis-like conditions, and it seems insufficient to support the diagnosis of $\mathrm{AC}$ when used alone.

Connell et al. ${ }^{10}$ did not provide numerical measurements, and they stated that the SGHL thickness increased and showed signal changes in patients diagnosed with
AC. Petchprapa et al. ${ }^{8}$ reported that the SGHL thickness increased in AC cases, although the amount of thickening was not quantitatively specified. In this study, SGHL thickness was evaluated in the diagnosis of $\mathrm{AC}$, and the AUC value was calculated as 0.972 when a $2-\mathrm{mm}$ cutoff was accepted according to the median value. The results we obtained from this study show that an SGHL thickness of $2 \mathrm{~mm}$ and above can help diagnose AC.

The measurement of IGHL thickness is another valuable method in the diagnosis of AC. Teixeira et al. ${ }^{11}$ observed that signal enhancement in IGHL on T2 sequences had high sensitivity and specificity. Zappia et al. ${ }^{12}$ showed an increase in IGHL signal with high sensitivity and specificity in T2W sequences in patients diagnosed with AC. Bang et al. ${ }^{13}$ measured the mean IGHL anterior band thickness as $4.52 \pm 1.02 \mathrm{~mm}$ for the $54 \mathrm{AC}$ patients and $3.47 \pm 0.99 \mathrm{~mm}$ for the control group. When the information obtained from this study and the information obtained from similar studies are evaluated together, an IGHL value of $4 \mathrm{~mm}$ and above can be considered as a favorable criterion for the diagnosis of AC.

Emig et al. ${ }^{14}$ suggested that there was no statistically significant difference between the AC patients and the control group in terms of the CHL thickness measured on conventional shoulder MRI. In a more recent conventional shoulder MRI study, Li et al. ${ }^{15}$ measured the CHL thickness as $3.99 \pm 1.68 \mathrm{~mm}$ for the AC group and $3.08 \pm 1.32 \mathrm{~mm}$ for the control group. In this study, the CHL thickness was measured as $4.66 \pm 1.13(1-8) \mathrm{mm}$, and the difference between the two groups was statistically significant $(\mathrm{p}<0.05)$. According to the data obtained, CHL being over $4.6 \mathrm{~mm}$ can be considered as a finding supporting the diagnosis of AC.

As far as we know, although there are current studies examining signal changes in the RI, no quantitative studies are investigating soft-tissue thickness in the $\mathrm{RI}^{16,17}$. In this study, the soft-tissue thickness of the RI was found to be $3.72 \pm 2.24(0-9) \mathrm{mm}$ in the patients with $\mathrm{AC}$, and the difference between the two groups being statistically significant $(\mathrm{p}<0.05)$. This result is a statistically significant value and shows that the soft-tissue thickness in the RI must be evaluated in shoulder MR examination for the diagnosis of AC. There is a need for an increase in large series of studies on this subject.

The limitations of the study include the retrospective evaluation of the shoulder MRI examinations and the study being conducted in a single center. Another limitation can be considered as the inability to histopathologically confirm the presence of inflammation due to the retrospective study design. 


\section{CONCLUSIONS}

Clinicians frequently refer to shoulder MRI to confirm the diagnosis of AC and exclude other possible diagnoses. For this reason, there is a need to clarify the diagnostic criteria for AC. According to the data obtained in this study, the thickness of SGHL over $2 \mathrm{~mm}$ or the soft-tissue thickness in the RI of 3.6 and above can be used to support the diagnosis of AC diagnosis. In addition, CHL thickness of $4.6 \mathrm{~mm}$ and above and the thickness of IGHL over $4 \mathrm{~mm}$ can be counted among the criteria that support the diagnosis of AC.

\section{AUTHORS' CONTRIBUTIONS}

HA: Conceptualization, Methodology, Validation, Software, Writing -original draft. ES: Resources, Investigation, Formal analysis, Validation, Writing -original draft. OD: Project administration, Supervision, Visualization, Investigation. GS: Data curation, Methodology, Software, Supervision, Writing -original draft. BG: Methodology, Resources, Supervision, Visualization, Writing-review \& editing.

\section{REFERENCES}

1. Akpancar S, Örsçelik A, Seven MM, Koca K. The effectiveness of prolotherapy on failed rotator cuff repair surgery. Turk J Phys Med Rehabil. 2019;65(4):394-401. https://doi.org/10.5606/ tftrd.2019.3222

2. Park J, Choi YH, Chai JW, Cha SW, Lim JH, Jo CH, et al. Anterior capsular abnormality: another important MRI finding for the diagnosis of adhesive capsulitis of the shoulder. Skeletal Radiol. 2019;48(4):543-52. https://doi.org/10.1007/s00256018-3064-8

3. D'Orsi GM, Via AG, Frizziero A, Oliva F. Treatment of adhesive capsulitis: a review. Muscles Ligaments Tendons J. 2012;2(2):70-8. PMID: 23738277

4. Manske RC, Prohaska D. Diagnosis and management of adhesive capsulitis. Curr Rev Musculoskelet Med. 2008;1(34):180-9. https://doi.org/10.1007/s12178-008-9031-6

5. Mengiardi B, Pfirrmann CWA, Gerber C, Hodler J, Zanetti M. Frozen shoulder: MR arthrographic findings. Radiology. 2004;233(2):486-92. https://doi.org/10.1148/radiol.2332031219

6. DeLong ER, DeLong DM, Clarke-Pearson DL. Comparing the areas under two or more correlated receiver operating characteristic curves: a nonparametric approach. Biometrics. 1988;44(3):837-45. PMID: 3203132

7. Gokalp G, Algin O, Yildirim N, Yazici Z. Adhesive capsulitis: contrast-enhanced shoulder MRI findings. J Med Imaging Radiat Oncol. 2011;55(2):119-25. https://doi.org/10.1111/j.17549485.2010.02215.x

8. Petchprapa CN, Beltran LS, Jazrawi LM, Kwon YW, Babb JS. The rotator interval: a review of anatomy, function, and normal and abnormal MRI appearance. AJR Am J Roentgnol. 2010;195(3):567-76. https://doi.org/10.2214/AJR.10.4406.

9. Sofka CM, Ciavarra GA, Hannafin JA, Cordasco FA, Potter HG. Magnetic resonance imaging of adhesive capsulitis: correlation with clinical staging. HSS J. 2008;4(2):164-9. https://doi.org/10.1007/s11420-008-9088-1
10. Connell D, Padmanabhan R, Buchbinder R. Adhesive capsulitis: role of MR imaging in differential diagnosis. Eur Radiol. 2002;12(8):2100-6. https://doi.org/10.1007/s00330-0021349-7

11. Teixeira PAG, Balaj C, Chanson A, Lecocq S, Louis M, Blum A. Adhesive capsulitis of the shoulder: value of inferior glenohumeral ligament signal changes on T2-weighted fat-saturated images. AJR AM J Roentgenol. 2012;198(6):W58996. https://doi.org/10.2214/AJR.11.7453

12. Zappia M, Di Pietto F, Aliprandi A, Pozza S, De Petro P, Muda $A$, et al. Multi-modal imaging of adhesive capsulitis of the shoulder. Insights Imaging. 2016;7(3):365-71. https://doi. org/10.1007/s13244-016-0491-8

13. Bang YS, Park J, Lee SY, Park J, Park S, Joo Y, et al. Value of anterior band of the inferior glenohumeral ligament area as a morphological parameter of adhesive capsulitis. Pain Res Manag. 2019;2019:9301970. https://doi.org/10.1155/2019/9301970

14. Emig EW, Schweitzer ME, Karasick D, Lubowitz J. Adhesive capsulitis of the shoulder: MR diagnosis. AJR Am J Roentgenol. 1995;164(6):1457-59. https://doi.org/10.2214/ ajr.164.6.7754892

15. Li JQ, Tang KI, Wang J, Li QY, Xu HT, Yang HF, et al. MRI Findings for frozen shoulder evaluation: is the thickness of the coracohumeral ligament a valuable diagnostic tool? Plos One. 2011;6(12):e28704. https://doi.org/10.1371/journal. pone.0028704

16. Zhao W, Zheng $X$, Liu $Y$, Yang W, Amirbekian V, Diaz LE, et al. An MRI study of symptomatic adhesive capsulitis. PLoS One. 2012;7(10):e47277. https://doi.org/10.1371/journal. pone.0047277

17. Pessis E, Mihoubi F, Feydy A, Campagna R, Guerini H, Roren $A$, et al. Usefulness of intravenous contrast-enhanced MRI for diagnosis of adhesive capsulitis. Eur Radiol. 2020;30(11):598191. https://doi.org/10.1007/s00330-020-07003-4 